\title{
A Type-2 Fuzzy Method for Identification of Disease-related Genes on Microarrays
}

\author{
Yan-Fei Wang, Zu-Guo Yu
}

\begin{abstract}
Fuzzy set theory has been widely used in the analysis of gene microarray data. However, due to noise and uncertainty inherent in microarray data, traditional fuzzy methods sometimes do not perform well. In this paper, we propose a type-2 fuzzy membership test (Type-2 FM test) for disease-associated gene identification on microarrays to improve traditional fuzzy methods. We apply this method on diabetes and lung cancer microarrays and make a comparison with traditional fuzzy methods. For diabetes data, we can identify 7 genes which have been confirmed to be related to diabetes treatment in the published literature and one more gene can be identified than original approaches. For lung cancer data, we can also identify 7 genes which have been confirmed to be associated with lung cancer treatment in published literature and the type- 2 d-values are significantly different. The results show that our type-2 FM test performs better than traditional fuzzy methods when analyzing microarray data with similar expression values and noise.
\end{abstract}

Keyworks-type-2 fuzzy set; membership; microarray data; uncertainties.

\section{INTRODUCTION}

Microarray technology has been used in many areas of bioinformatics research. It can monitor expression of thousands of genes in parallel. The enormous quantities of information data generated have led to a great demand for efficient analysis methods. For some genes, the expression values are different between patients and normal people. Making comparison of these gene expression data in patients and those of normal people can identify disease-associated genes and enhance our understanding of diseases [1]. Researchers have made significant breakthroughs and progress in analysis of disease gene microarray data. Most importantly, they also have identified many genes that have relationship with onset, development and progression of various diseases $[2,3]$.

In order to identify the disease-associated genes, we usually

Manuscript received March 18, 2011. This project was supported by the Australian Research Council (Grant No. DP0559807), the Natural Science Foundation of China (Grant No. 11071282), the Chinese Program for New Century Excellent Talents in University (Grant No. NCET-08006867), the Lotus Scholars Program of Hunan province of China, the Aid program for Science and Technology Innovative Research Team in Higher Educational Institutions of Hunan Province of China and a China Scholarship Council-Queensland University of Technology Joint Scholarship.

Yan-Fei Wang is a $\mathrm{PhD}$ candidate in applied mathematics. He is now studying in Discipline of Mathematical Sciences, Queensland University of Technology, Brisbane, QLD, 4001 Australia (e-mail: yfmu@sina.com)

$\mathrm{Zu}-\mathrm{Guo} \mathrm{Yu}$ is a Professor in Mathematics at Xiangtan University of China and a Research Fellow in Mathematics at Queensland University of Technology, Australia. (corresponding to: 086-731-58298760; fax: 086-731-58293934; e-mail:yuzg@hotmail.com). need to determine for each gene whether the two sets of expression values are significantly different from each other. Measuring the divergence of two sets of values of gene expression data is an effective approach. Two commonly-used statistical methods to measure the divergence of two sets of values are the t-test and the Wilcoxon rank sum test [4]. However, both methods have limitations. The t-test is very sensitive to extreme values and cannot distinguish two sets with close means even though the two sets are significantly different form each other. Although the rank sum test overcomes the limitation of the t-test in sensitivity to extreme values, it is not sensitive to absolute values. This might be advantageous to some applications but not to others [1].

DNA microarray data contains uncertainty and noise $[5,6]$. The above two methods cannot manage this uncertainty properly and always obtains poor quality results [1]. The word "different" itself is a fuzzy concept and fuzzy theory has many advantages in dealing with data containing uncertainty; therefore fuzzy approaches have been taken into consideration to analyze DNA microarrays. Liang et al. [1] proposed a fuzzy set theory based approach, namely a fuzzy membership test (FM-test), for disease genes identification and obtained better results by applying their approach on diabetes and lung cancer microarrays. However, some limitations still exist. The most obviously one is when the values of gene microarray data are very similar and lack of over-expression, in which case the FM-d values are very close or even equal to each other. That made the FM-test inadequate in distinguishing disease genes [1]. Meanwhile, DNA microarray data contains noise, hence yielding uncertain information in the original data. When deriving the membership function for evaluation, all of these uncertainties translate into uncertainties about fuzzy set membership function. Type-1 fuzzy sets are not able to directly model such uncertainties because their membership functions are totally crisp [7].

To overcome these problems, we introduced type-2 fuzzy set theory into the research of disease-associated gene identification. Type- 2 fuzzy sets can control the uncertainty information more effectively than conventional type-1 fuzzy sets because the membership functions of type-2 fuzzy sets are three-dimensional. It is the new third dimension of type-2 fuzzy sets that provides additional degrees of freedom that makes it possible to directly model uncertainties [7].

In this paper we establish the type-2 fuzzy membership function for identification of disease-associated genes on microarray data of patients and normal people and apply the type-2 FM-test of this method to diabetes and lung cancer data. For the ten best-ranked genes of diabetes identified by the type-2 FM-test, 7 of them have been confirmed as diabetes associated genes according to genes description information in Genebank and the published literature. One more gene than original approaches is identified. Within the 10 best ranked 
genes identified in lung cancer data, 7 of them are confirmed by the literature which is associated with lung cancer treatment. The type-2 FM-d values are significantly different, which makes the identifications more reasonable and convincing than the original FM-test.

\section{TYPE-2 FUZZY SETS}

\section{A. Overview of Type-2 Fuzzy Set}

Type-2 fuzzy set was introduced as an extension of a type-1 fuzzy set by Zadeh [8]. Mizumoto and Tanaka [9] studied the set theoretic operations and properties of membership grades of such sets and examined their operations of algebraic product and algebraic sum. Karnik and Mendel [10] extended the algorithms for performing union, intersection and complement for type-2 fuzzy sets. Type-2 fuzzy sets and tools have now been widely used in many fields.

In this paper, we use the following notation and terminology: a type- 2 fuzzy set in the universal set $X$, denoted as $\tilde{A}$, can be characterized by a type- 2 fuzzy membership function $\mu_{\tilde{A}}(x, u)$ as

$$
\tilde{A}=\int_{x \in X} \mu_{\tilde{A}}(x) / x=\int_{x \in X}\left[\int_{u \in J_{x}} f_{x}(u) / u\right] / x, J_{x} \subseteq[0,1],
$$

where $\mu_{\tilde{A}}(x)$ is the membership grade of $x \in X$, which is a type- 1 fuzzy set in $[0,1] ; J_{x}$ is the primary membership of $x$, a memberships of the primary membership of $x$ is called a secondary membership of $x$ in $\tilde{A}$, denoted as $f_{x}(u)$. Fig 1 shows an example of a type-2 fuzzy membership function. The uncertainty in the primary membership, consisting of a bounded region by upper and lower membership functions is called the footprint of uncertainty (FOU) which can be expressed as

$$
\operatorname{FOU}(\tilde{A})=\bigcup_{\forall x \in X} J_{x}=\left\{(x, u): u \in J_{x} \subseteq[0,1]\right\} .
$$

Although type-2 fuzzy sets can be useful in modeling uncertainty where type-1 fuzzy sets cannot, the operations of type-2 fuzzy sets involve numerous embedded type-2 fuzzy sets which include all possible combinations of secondary membership values. Therefore, a large number of computations are required. However, interval type-2 fuzzy sets can reduce the computational complexity significantly [11]. Due to this property, we apply the interval type-2 fuzzy set instead of a general type-2 fuzzy set in this paper. An interval type-2 fuzzy set is a specific type-2 fuzzy set when $f_{x}(u)=1, \forall u \in J_{x} \subseteq[0,1]$, and is expressed as

$$
\tilde{A}=\int_{x \in X}\left[\int_{u \in J_{x}} 1 / u\right] / x
$$

Fig. 2 shows the secondary membership function of an interval type-2 fuzzy set at $x=4$, in which the secondary memberships are all equally weighted for each primary membership of $x=4$. Therefore, $J_{x}$ can be expressed as

$$
J_{x}=\left\{(x, u): u \in\left[\underline{u}_{\tilde{A}}(x), \bar{u}_{\tilde{A}}(x)\right]\right\}
$$

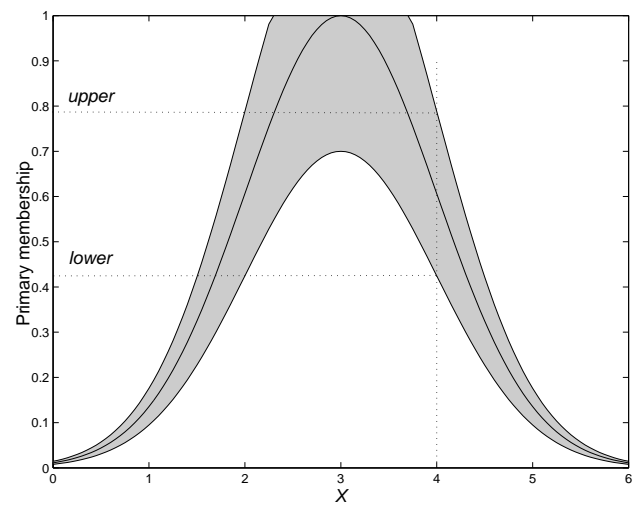

Figure 1. Illustration of a type-2 fuzzy membership function

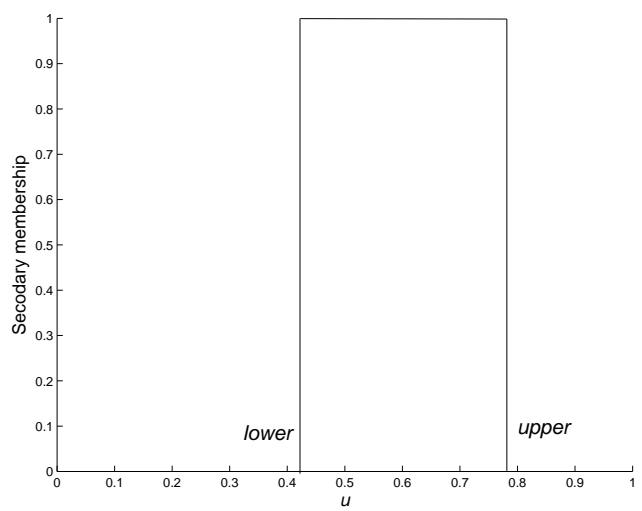

Figure 2. Interval secondary membership function

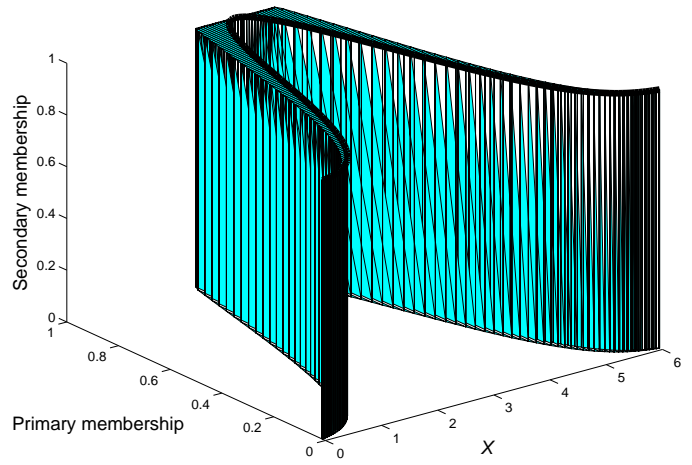

Figure 3. 3D illustration of type-2 fuzzy sets

Where $\underline{u}_{\tilde{A}}(x)$ denote as lower bound of FOU and $\bar{u}_{\tilde{A}}(x)$ denote as upper bound of FOU. Moreover, FOU in (2) can be expressed as

$$
\left.\operatorname{FOU}(\tilde{A})=\bigcup_{\forall x \in X}\left[\underline{u}_{\tilde{A}}(x), \bar{u}_{\tilde{A}}(x)\right]\right\}
$$

Fig. 3 is a $3 \mathrm{D}$ plot of a type-2 fuzzy set whose secondary membership is a triangular function. As shown in the figure, the membership function of type-2 fuzzy sets is three-dimensional.

\section{B Type-2 Fuzzy Membership function Generation}

In this paper, the method we used for type-2 fuzzy membership function generation is heuristic method. The heuristic method usually used an appropriate predefined type-1 fuzzy membership function, such as triangular, 
trapezoidal, Gaussian, $\mathrm{S}$, or $\pi$ function to describe the distribution of the pattern data [11]. Some frequently used heuristic membership functions are as follows.

Triangular function:

$$
\mu(x ; a, b, c)=\left\{\begin{array}{cl}
0 & \text { if } \quad x \leq a \\
\frac{x-a}{b-a} & \text { if } a \leq x \leq b \\
\frac{c-x}{c-b} & \text { if } b \leq x \leq c \\
0 & \text { if } \quad x \geq c
\end{array} .\right.
$$

Trapezoidal function

$$
\mu(x ; a, b, c)=\left\{\begin{array}{clr}
0 & \text { if } \quad x \leq a \\
\frac{x-a}{b-a} & \text { if } a \leq x \leq b \\
1 & \text { if } b \leq x \leq c \\
\frac{c-x}{c-b} & \text { if } c \leq x \leq d \\
0 & \text { if } \quad x \geq d
\end{array}\right.
$$

Guassian function

$$
\mu(x)=e^{\frac{-(x-\mu)^{2}}{2 \sigma^{2}}}
$$

S function

$$
S(x ; a, b, c)=\left\{\begin{array}{ccc}
0 & \text { if } & x \leq a \\
2 \cdot\left(\frac{x-a}{c-a}\right)^{2} & \text { if } & a<x \leq b \\
1-2 \cdot\left(\frac{x-a}{c-a}\right)^{2} & \text { if } & b<x \leq c \\
1 & \text { if } & x>c
\end{array},\right.
$$

where $b=\frac{a+c}{2}$.

\section{$\pi$ function}

$$
\Pi(x ; a, b, c)=\left\{\begin{array}{c}
S\left(x ; c-b, c-\frac{b}{2}, c\right) \quad x \leq c \\
1-S\left(x ; c, c+\frac{b}{2}, c+b\right) x<c
\end{array} .\right.
$$

After a type-1 fuzzy membership function is selected for a given data set, we need determine upper and lower membership function. Usually the original type-1 fuzzy membership function is used as the upper bound and a parameter $\alpha$ between 0 and 1 is set to obtain the lower bound. The parameter $\alpha$ can also be provided by experts. The FOU of our heuristic method can be expressed as

$$
\begin{aligned}
& \bigcup_{\forall x \subset X}[\underline{\mu}(x), \bar{\mu}(x)]= \\
& \bigcup_{\forall x \subset X}[\mu(x), \alpha \cdot \mu(x)], \quad 0<\alpha<1
\end{aligned}
$$

The heuristic method is summarized as follows:

(i) Select a heuristic type-1 fuzzy membership function which is suitable for a given data set.

(ii) Set the parameter for the membership function which can be also provided by an expert.

\section{Type Reduction}

In a type-1 fuzzy logic system, the output corresponding to each rule is a type- 1 set in the output space. To obtain the final result, defuzzification is needed. For type-2 fuzzy logic system, this step is considered as "type reduction" [11]. According to type reduction, a type-2 fuzzy set turns into a traditional fuzzy set and then output a crisp number to represent the combined output e.g., the centroid defuzzifier finds the union of all the out put sets and uses the centroid of the union as the crisp output. The traditional method to obtain centroid of a type-1 $\operatorname{set} A$, whose domain is discretized into $\mathrm{N}$ points is given as

$$
C_{A}=\frac{\sum_{i=1}^{N} x_{i} \mu_{A}\left(x_{i}\right)}{\sum_{i=1}^{N} \mu_{A}\left(x_{i}\right)}
$$

However, in this paper, what we need is just the membership of each gene and we do not need the crisp output. Therefore, we just calculate the primary and secondary membership values and combine them into traditional membership, then rank the genes based on them. The detail of type reduction would be described in next section.

\section{METHODS}

In this section, based on the type-2 fuzzy set theory and the work of Liang et. al [1], we propose an interval type-2 FM-test for disease-associated gene identification. For each gene, the microarray data contain expression values from both patients and normal people. Table I. provides an example which contains expression values of 3 genes for two groups of people that are associated with diabetes: five insulin-sensitive (IS) people and five insulin-resistant (IR) people. We denote by $S_{1}$ the value set of IS and by $S_{2}$ the value set of IR. We consider $S_{1}$ and $S_{2}$ as two samples of a type- 2 fuzzy set. The average membership value gives a measure of the divergence of the original two sets. In particular, this method is performed as follows:

TABLE I. Expression values of IR and IS people

\begin{tabular}{|c|c|c|c|c|c|c|c|c|c|c|}
\hline Gene & \multicolumn{9}{|c|}{ IR } & \multicolumn{5}{|c|}{ IS } \\
\hline 1 & 123 & 142 & 11 & 406 & 220 & 305 & 398 & 707 & 905 & 688 \\
\hline 2 & 200 & 191 & 220 & 83 & 197 & 49 & 81 & 116 & 111 & 135 \\
\hline 3 & 598 & 424 & 695 & 451 & 141 & 342 & 260 & 266 & 229 & 234 \\
\hline
\end{tabular}


1. Using the Gaussian function as the primary membership function to compute the mean $\left(\mu_{1}, \mu_{2}\right)$ and standard deviation $\left(\sigma_{1}, \sigma_{2}\right)$ of $S_{1}$ and $S_{2}$.

2. Establishing the primary membership function. The means for the upper and lower primary membership functions are obtain as

$$
\mu_{\text {upper }}=\max \left(\mu_{1}, \mu_{2}\right), \mu_{\text {lower }}=\min \left(\mu_{1}, \mu_{2}\right)
$$

We can obtain the standard deviations for the upper and lower primary membership function in the same way.

3. Using the primary membership function $J_{\text {upper }}(x)$, $J_{\text {lower }}(x)$, and the secondary membership values $f_{x}(u)$ to quantify the convergence of $S_{1}$ and $S_{2}$. Type-reduction work is needed in this step. Here, because we use the interval type- 2 fuzzy set, $f_{x}(u)=1, \forall u \in J_{x} \subseteq[0,1]$. The secondary memberships are all uniformly weighted for each primary membership of $x$.

4. Calculating the divergence degree between the two sets based on the convergence degree.

Type reduction is an important step for type-2 fuzzy sets [11]. $\forall x \in X$, a primary membership interval [ $J_{\text {lower }}(x)$, $\left.J_{\text {upper }}(x)\right]$ can be obtained. We discretize it into $N$ points, where $a_{1}=J_{\text {lower }}(x)$ and $a_{N}=J_{\text {upper }}(x)$; then the final membership of $x$ can be obtain as

$$
\mu(x)=\frac{\sum_{i=1}^{N} a_{i} * f_{x}\left(a_{i}\right)}{N}
$$

This type reduced membership $\mu(x)$ maps each value $x$ in $S_{1}$ or $S_{2}$ into a membership value to quantify the degree that $\mathrm{x}$ belongs to a type- 2 fuzzy set $\mu_{\tilde{A}}(x)$. For simplicity, we put $a_{i}=$ $\left(a_{i-1}+a_{i+1}\right) / 2, i=2, \ldots, N$-1, while $f_{x}\left(a_{i}\right)=1 . \forall x \in X, i=$ $1, \ldots, N$, Eq. (14) can be expressed as

$$
\mu(x)=\frac{J_{\text {lower }}(x)+J_{\text {upper }}(x)}{2}
$$

Because the membership function maps the elements of $S_{1}$ and $S_{2}$ into the same type-2 fuzzy set, the aggregation of all membership values in the two sets can be used to quantify the similarity of $S_{1}$ and $S_{2}$. It can be considered as an overall bond between these two sets. The weaker this overall bond is, the more divergent these two sets are. In this case, for a given gene, the expression values between patients and normal people can be significantly different. If the elements in both $S_{1}$ and $S_{2}$ have high membership values, $S_{1}$ is very similar with $S_{2}$. However when they both have low values we cannot say $S_{1}$ and $S_{2}$ are similar, because the type-2 fuzzy set is established based on both $S_{1}$ and $S_{2}$. If the elements in $S_{1}$ have low membership values, the reason may be the effect from elements in $S_{2}$. So if the elements in the two sets all have low values, it also suggests that $S_{1}$ and $S_{2}$ are different.

To compute the overall bond between $S_{1}$ and $S_{2}$, we define type-2 FM c-values based on Liang et al. [1]:

Definition 1 Given two sets $S_{1}$ and $S_{2}$, the convergence degree between $S_{1}$ and $S_{2}$ is defined as

$$
c\left(S_{1}, S_{2}\right)=\frac{\sum_{x \in S_{1}} \mu(x)+\sum_{y \in S_{2}} \mu(y)}{\left|S_{1}\right|+\left|S_{2}\right|}
$$

We define the divergence value as follows:

Definition 2 Given two sets $S_{1}$ and $S_{2}$, the divergence degree between $S_{1}$ and $S_{2}$ is defined as

$$
d\left(S_{1}, S_{2}\right)=1-c\left(S_{1}, S_{2}\right)
$$

\section{RESUlts AND DisCUSSION}

We use two different data sets from the literature. The first data set is the Diabetes data [12]. The original data set contains 10831 genes. To make this data more reliable, only the genes with no null expression values are used for analysis. There are ten expression values in each gene, five from insulin-sensitive (IS) people and the other five from insulin-resistant (IR) people. The second data set is lung cancer data which contain 22283 genes [13]. The first five values of each gene in this data set are from squamous lung cancer biopsy specimens and the others are from paired normal specimens. We use the method described above and Liang's FM-test approach on these data sets, and then make a comparison on these two methods. In Tables II and III, the bold letters are names of identified genes.

\section{A. Analysis of Diabetes dataset}

Ten best-ranked genes of diabetes identified by the type- 2 FM-test and the original FM-test are shown in Table II. From this table we see that the results of the two methods are not much different. We identified gene U61734 and gene Z26491 by the type-2 FM-test instead of gene U06452 and gene X57959 identified by the FM-test. Gene U06452 and gene $X 57959$ are just candidate genes for diabetes mentioned in some published papers, but no biological evidence was put forward to confirm that they are related with diabetes $[1,12]$. However, for gene Z26491, the homo sapiens gene for catechol o-methyltrans-fease (COMT), was found to be differently expressed and helpful for treatment in diabetic rats. Ref. [14] compared the activity of COMT in the livers of diabetic rats with that in normal rats; the result suggested the activity of COMT is lower in diabetic rats than in normal rats. Lal et al [15] examined the effect of nitecapone, an inhibitor of the dopamine-metabolizing enzyme COMT and a potent antioxidant, on functional and cellular determinants of renal function in rats with diabetes. The results suggested that the COMT inhibitory and antioxidant properties of nitecapone provide a protective therapy against the development of diabetic nephropathy [15]. These works proved that gene $Z 26491$ is related with diabetes or treatment. In the remaining genes, U45973, M60858, L07648, L07033, X53586, X81003 are diabetes associated genes according to the current literature [1]. D85181, M95610 and U06452 are recommended as candidate diabetes genes by Liang et al. [15], while $X 57959$ was recommended in Yang et al. [12]. We recommended gene $U 61734$ as candidate gene for future research in this field. From the comparison, one more disease-associated gene is identified by the type-2 FM-test. 
TABLE II. TEN BEST-RANKED GENES ASSOCIATED WITH DIABETES IDENTIFIED BY THE TWO METHODS.

\begin{tabular}{|c|c|c|}
\hline \multicolumn{3}{|c|}{ Type-2 FM-test } \\
\hline Probe Set & Gene Description & T2 d-value \\
\hline $\mathbf{U 4 9 5 7 3}$ & $\begin{array}{c}\text { Human phosphatidylinositol (4,5) } \\
\text { bisphosphate }\end{array}$ & 0.6733 \\
\hline $\mathbf{X 5 3 5 8 6}$ & Human. mRNA for integrin alpha 6 & 0.6131 \\
\hline $\mathbf{M 6 0 8 5 8}$ & Human. nucleolin gene & 0.6080 \\
\hline U61734 & $\begin{array}{c}\text { Homo sapiens transmembrane emp24-like } \\
\text { trafficking protein 10 }\end{array}$ & 0.5831 \\
\hline D85181 & $\begin{array}{c}\text { Homo sapiens mRNA for fungal } \\
\text { sterol-C5-desaturase homolog }\end{array}$ & 0.5808 \\
\hline $\mathbf{Z 2 6 4 9 1}$ & $\begin{array}{c}\text { Homo sapiens gene for catechol } \\
\text { o-methyltrans-fease }\end{array}$ & 0.5773 \\
\hline $\mathbf{L 0 7 6 4 8}$ & Human MXII mRNA & 0.5769 \\
\hline M95610 & $\begin{array}{c}\text { Human alpha 2 type IX collagen (COL9A2) } \\
\text { mRNA }\end{array}$ & 0.5760 \\
\hline $\mathbf{L 0 7 0 3 3}$ & $\begin{array}{c}\text { Human hydroxymethylglutaryl-CoA lyase } \\
\text { mRNA }\end{array}$ & 0.5749 \\
\hline $\mathbf{X 8 1 0 0 3}$ & Homo sapiens HCG V mRNA & 0.5525 \\
\hline \multicolumn{3}{|c|}{ FM-test } \\
\hline Probe Set & Gene Description \\
\hline $\mathbf{U 4 5 9 7 3}$ & Human phosphatidylinostiol (4,5) \\
bisphosphate & FM d-value \\
\hline M60858 & Human nucleolin gene & 0.9988 \\
\hline D85181 & $\begin{array}{c}\text { Homo sapiens mRNA for fungal } \\
\text { sterol-C5-desaturase homolog }\end{array}$ & 0.9351 \\
\hline M95610 & $\begin{array}{c}\text { Huamn alpha 2 type IX collagen (COL9A2) } \\
\text { mRNA }\end{array}$ & 0.8918 \\
\hline $\mathbf{L 0 7 6 4 8}$ & Human MXII mRNA & 0.8718 \\
\hline $\mathbf{L 0 7 0 3 3}$ & Human hydroxymethylglutaryl-CoA lyase \\
mRNA & 0.8554 \\
\hline $\mathbf{X 5 3 5 8 6}$ & Human mRNA for integrin alpha 6 & 0.8513 \\
\hline $\mathbf{X 8 1 0 0 3}$ & Homo sapiens HCG V mRNA & 0.7914 \\
\hline X57959 & Ribosomal protein L7 & 0.7676 \\
\hline $\mathbf{4 0 6 4 5 2}$ & Melan-A & 0.7566 \\
\hline
\end{tabular}

\section{B. Analysis of lung cancer data}

Table III shows ten best-ranked genes of lung cancer identified by the two approaches. The results are very different. In the result obtained by the type- 2 FM-test, 7 disease associated genes are identified. MFNG is a member of the fringe gene family which also includes radical and lunatic fringe genes. They all encode evolutionarily conserved secreted proteins that act in the Notch receptor pathway. The activity of fringe proteins can alter Notch signaling [16]. Activation of the Notch 1 signaling pathway can impair small cell lung cancer viability [17]. The protein encoded by CTSW is found associated with the membrane inside the endoplasmic reticulum of natural killer (NK) [16]. NK cells play a major role in the rejection of tumors and cells infected by viruses [18]. ALDH3B1 is highly expressed in kidney and lung [16]. Marchitti et al. [19] found ALDH3B1 expression was upregulated in a high percentage of human tumors; particularly in lung cancer cell the value is highest. Increasing ALDH3B1 expression in tumor cells may confirm a growth advantage or be the result of an induction mechanism mediated by increasing oxidative stress [19]. LPCAT1 activity is required to achieve the levels of SatPC essential for the transition to air breathing [20] and it is also upregulated in cancerous lung [21]. Gene PDE3B was mentioned in [22] as the most significantly amplified gene in the tumors. CITED2 is required for fetal lung maturation [23]. Researchers found CITED2 was highly expressed in lung cancer but not in normal tissues, which demonstrates that CITED2 plays a key role in lung cancer progression [24]. Gene DLC1 encodes protein deleted in liver cancer [1]. This gene is deleted in the primary tumor of hepatocellular carcinoma. It maps to $8 \mathrm{p} 22-\mathrm{p} 21.3$, a region frequently deleted in solid tumors. It is suggested that this gene is a tumor suppressor gene for human liver cancer, as well as for prostate, lung, colorectal and breast cancers [16].

8 disease-associated genes are identified by the original FM-test method [1]. However, when we applied the FM-test on lung cancer data, there are more than 80 genes having the same FM d-values; they are all equal to one, which made it difficult to rank and distinguish disease associated genes from others. We have to choose the overexpressed genes from these 80 genes for analysis, which made the task more complicated, and it may miss some important genes. The reason is that the gene expression values in lung cancer microarray data are very close to each other, and the original data is noisy. The FM-test does not seem to be able to deal with these factors suitably.

TABLE III. Ten best-ranked genes associated with lung cancer.

\begin{tabular}{|c|c|c|}
\hline \multicolumn{3}{|c|}{ Type-2 FM-test } \\
\hline Probe Set & Gene Description & T2 d-value \\
\hline NM_002405 & $\begin{array}{c}\text { MFNG: MFNG O-fucosylpeptide } \\
\text { 3-beta-N-acetylglucosaminyltransferase }\end{array}$ & 0.7435 \\
\hline NM 001335 & CTSW: cathepsin W & 0.7285 \\
\hline NM_017761 & $\begin{array}{l}\text { PNRC2: praline-rich nuclear receptor } \\
\text { coactivator } 2\end{array}$ & 0.7266 \\
\hline AV728526 & DTX4: deltex homolog 4 (Drosophila) & 0.7265 \\
\hline NM_0002694 & $\begin{array}{l}\text { ALDH3B1: aldehyde dehydrogenase } 3 \\
\text { family, member B1 }\end{array}$ & 0.7259 \\
\hline NM_024830 & $\begin{array}{c}\text { LPCAT1: lysophosphatidylcholine } \\
\text { acyltransferase } 1\end{array}$ & 0.7243 \\
\hline BE789881 & RAB31: member RAS oncogene family & 0.7204 \\
\hline AA888858 & $\begin{array}{l}\text { PDE3B: phosphodiesterase 3B, } \\
\text { cGMP-inhibited }\end{array}$ & 0.7194 \\
\hline NM_006079 & $\begin{array}{l}\text { CITED2: cbp/p300-interacting } \\
\text { transactivator, with Glu/Asp-rich } \\
\text { carboxy-terminal domain,2 }\end{array}$ & 0.7186 \\
\hline AF026219 & DLC1:deleted in liver cancer 1 & 0.7145 \\
\hline \multicolumn{3}{|c|}{ FM-test (Overexpressed) } \\
\hline Probe Set & Gene Description & FM d-value \\
\hline NM_173086 & KRT6E: Keratin 6E & 1 \\
\hline NM 001723 & DST: Dystonin & 1 \\
\hline NM_002639 & $\begin{array}{l}\text { SERPINB5: Serpin peptidase inhibitor, } \\
\text { clade B (ovalbumin), member } 5\end{array}$ & 1 \\
\hline AB010153 & TP73L: Tumor protein p73 like & 1 \\
\hline NM 023915 & GPR87: G protein-coupled receptor 87 & 1 \\
\hline NM_006536 & $\begin{array}{c}\text { CLCA2: Chloride channel, calcium } \\
\text { activated, family member } 2\end{array}$ & 1 \\
\hline $\begin{array}{c}\text { NM_00100533 } \\
7\end{array}$ & $\begin{array}{l}\text { PKPI: Plakophilin 1 ( ectodermal } \\
\text { dysplasia/skin fragility syndrome) }\end{array}$ & 1 \\
\hline AF043977 & $\begin{array}{l}\text { CLCA2: Chloride channel, calcium } \\
\text { activated, family member } 2\end{array}$ & 1 \\
\hline NM_004415 & DSP: Desmoplakin & 1 \\
\hline NM_019093 & $\begin{array}{l}\text { UGTIA9: UDP glucuronosyltransferase ! } \\
\text { family, polypeptide A9 }\end{array}$ & 1 \\
\hline
\end{tabular}

\section{CONCLUSIONS}

We proposed a type-2 FM-test approach based on the type-2 fuzzy set theory and the work of Liang et al. [1]. This approach was then applied on diabetes and lung cancer microarray data. For each data set, we analyzed 10 best-ranked genes. In diabetes data, we identified 7 genes which have been confirmed to be related to diabetes or treatment in the published literature. In lung cancer data, we also identified 7 genes. The type- 2 FM d-values are significantly different for each gene, which makes the ranking more reasonable than from the original FM-test. The 
type-2 FM test performs better than the original FM test when analyzing microarray data containing similar expression values and noise.

\section{REFERENCES}

[1] L.R. Liang, S. Lu, X. Wang, Y. Lu, V. Mandal, D. Patacsil and D. Kumar, "FM-test: a fuzzy-set-theory-based approach to differential gene expression data analysis," BMC Bioinformatics, 7 (Suppl 4), 2006: S7, doi:10.1126/

[2] S. Rome , K. Clement, R. Rabasa-Lhoret, E. Loizon, C. Poitou, GS. Barsh, JP. Riou, M. Laville, H. Vidal, "Microarray profiling of human skeletal muscle reveals that insulin regulateds approximately 800 genes during a hyperinsulinemic clamp," J Biol Chem, vol. 278 (20), 2003, pp. 18063-18068.

[3] SE. Eckenrode, QG. Ruan, CD. Collins, P. Yang, RA. Mclndoe, A. Muir, JX. She, "Molecular pathways altered by insulin b9-23 immunization," Ann N Y Acad Sci, vol. 1037, 2004, pp: 175-185.

[4] B. Rosner, Fundamentals of Biostatistics. In Pacific Grove $5^{\mathrm{t}}$ editio n, CA: Duxbury Press, 2000.

[5] E. Someren, L. Wessels, M. Reindersa and E. Backer, "Regularization and noise injection for improving genetic newwork models," in Computational and statistical approaches to genomics ( $2^{\text {nd }}$ edition), W. Zhang and I. Shmulevich, Eds. Springer Science+Business Media, Inc, 2006, pp:279-295.

[6] J. Wang, K. R. Coombes, K. Baggerly, L. Hu, S. R. Hamilton, W. Wang, "Statistical considerations in the assessment of cDNA microarray data obtained using amplification," in Computation and statistical approaches to genomics, W. Zhang and I. Shmulevich, Eds. Springer Science+Business Media, Inc, 2006, pp: 21-36.

[7] J. M. Mendel and R. I. Bob John, "Type-2 fuzzy sets made simple”, IEEE Trans. On Fuzzy Systems, vol. 10, No. 2, pp: 117-128, April 2002.

[8] L. A. Zadeh, "The concept of a linguistic variable and its application to approximate reasoning-1," Inform. Sci., vol. 8, pp. 199-249, 1975.

[9] M. Mizumoto and K. Tanaka, "Some properties of fuzzy sets of type-2," Inform. Control, vol. 31, pp. 312-340, 1976.

[10] N. N. Karnik and J. M. Mendel, "Operations on type-2 fuzzy sets," Int. J. Fuzzy sets Syst., vol. 122, pp. 327-348, 2001.

[11] N. N. Karnik, J. M. Mendel, "Type-2 fuzzy logic systems," IEEE Trans on Fuzzy Systems, vol. 7, pp. 643-658, December, 1999.

[12] X. Yang, RT. Pratley, S. Tokraks, C. Bogardus, P. A. Permana, "Microarray profiling of skeletal muscle tissues from eqally obese, non-diabetic insulin-sensitive and insulin-resistant Pima Indians," Diabetologia, vol. 45 pp. 1584-1593, 2002.

[13] S. Wachi, K. Yoneda, R. Wu, "Interactome-transcriptone analysis reveals the high centrality of genes differentially expressed in lung cancer tissues." Bioinfromatics, Vol. 21, pp. 4205-4208, 2005.

[14] J. Wang, I. Liu, T. Tzeng, J. Cheng, "Decrease in catechol-omethyltransferase activity in the liver of streptozotocin-induced diabetic rats," Clin Exp Pharmacol \& Physiol, Vol. 29, pp. 419-422, May, 2002.

[15] M. A. Lal, A. Korner, Y. Matsuo. S. Zelenin, X. Cheng, G. Jarmko, G. DiBona, A. Eklof, A. Aperia, "Combined Antioxidant and COMT inhibitor treatment reverses renal abnormalities in diabetic rats," Diabetes, Vol. 49, pp.1381-1389, August, 2000.

[16] http://www.ncbi.nlm.nih.gov/genbank/.
[17] C. S. Platta, D. Y. Greenblatt. M. Kunnimalaiyaan, H. Chen, "Valproic acid induces Notch 1 signaling in small cell lung cancer cells," J. Surgical Research, Vol 148, pp. 31-37, July, 2008.

[18] R. Oldham, "Natural killer cells: Artifact to reality: an odyssey in biology," Cancer Metastasis Reviews, Vol. 2, pp. 323-326, 1983.

[19] A. S. Marchitti, D. J. Orlicky, C. Brocker, V. Vasiliou, “ Aldehyde Dehydrogenase 3B1 (ALD3B1): Immunohistochemical tissue distribution and cellular-specific localization in normal and cancerous human tissues," J. Histochemistry and Cytochemistry, Vol. 58, pp. 765-783, 2010.

[20] J. P. Bridges, M. Ikegami, L. L. Brilli, X. Chen, R. J. Mason, J. M. Shannon, "LPCAT1 regulates surfactant phospholipid synthese and is required for trasitioning to air breathing in mice," J. Clinical Investigation, vol. 120, 1736-1748, May, 2010.

[21] F. Mansillla, K. Costa, S. Wang, M. Kruhoffer, T. M. Lewin, T. F. Orntoft, R. A. Coleman, K. Birkenkamp-Demtroder, "Lysophosphatidylcholine acyltransferase 1 (LPCAT1) overexpression in human colorectal cancer," J. Mol Med, Vol. 87, pp. 85-97, January 2009.

[22] K. C. Lo, L. C. Stein, J. A. Panzarella, J. K. Cowell, L. Hawthorn, "Identification of genes involved in squamous cell carcinoma of the lung using synchronized data from DNA copy number and transcript expression profiling analysis," Lung Cancer, vol. 59, pp. 315-331, May, 2008.

[23] B. Xu, X. Qu, S. G, Y. Doughman, M. Watanabe, S. L. Dunwoodie, Y. Yang, "Cited2 is required for fetal lung maturation", Dev Biol, Vol. 317, pp. 95-105, May, 2008.

[24] Y. T. Chou, C.F. Hsu. Y. R. Kao, C. W. Wu, "Cited2, a novel EGFR-induced coactivator, plays a key role in lung cancer progression," AACR 101 ${ }^{\text {st }}$ Annual Meeting, Poster Presentation, 2010.

Yan-Fei, Wang was born in Jiaohe, Jilin, China, in 1983. He received both of the B.S. degree and M.S. degree in mathematics from Dalian Maritime University, Dalian, Liaoning, China in 2006 and 2008, respectively. His interests include fuzzy systems, fuzzy clustering, data mining and their applications to Bioinformatics.

$\mathrm{He}$ is currently a Ph.D. candidate in studying in Discipline of Mathematical Sciences, Queensland University of Technology, Brisbane, QLD, Australia.

Zu-Guo, Yu was born in Zhuzhou, China, in 1970. He received the B.S degree and M.S. degree in mathematics from Xiangtan University, Xiangtan, China in 1991 and 1994 respectively. He received the Ph.D degree in mathematics from Fudan University, Shanghai, China in 1997.

$\mathrm{He}$ is a Professor in Mathematics at Xiangtan University of China and a Research Fellow in Mathematics at Queensland University of Technology, Australia. He published almost 90 papers in many international journal such as Mol. Biol. Evol., BMC Evol. Biol., BMC Bioinformatics, J. Mol. Evol., J. Theor. Biol., J. Chem. Phys., J. Geophys. Res., Phys. Rev. E and Fuzzy sets a\& Systems. His current research focuses on fractals, bioinformatics, complex networks. 